\title{
Turn-on Loss Reduction for High Voltage Power Stack Using Active Gate Driving Method
}

\author{
Jin-Hong Kim*, Joon Sung Park*, Bon-Gwan Gu** and Chung-Yuen Won ${ }^{\dagger}$
}

\begin{abstract}
This paper presents an improved approach towards reducing the switching loss of insulated gate bipolar transistors (IGBTs) for a medium-capacity-class power conditioning system (PCS). In order to improve the switching performance, the switching operation is analyzed, and based on this analysis, an improved switching method that reduces the switching time and switching loss is proposed. Compared to a conventional gate drive scheme, the switching loss, switching time, and delay are improved in the proposed gate driving method. The performance of the proposed gate driving method is verified through several experiments.
\end{abstract}

Keywords: Active gate drive, Insulated Gate Bipolar Transistor (IGBT) gate driver, IGBT, Mediumvoltage drive

\section{Introduction}

Recently, the interest in renewable energy has increased because of environmental problems and high oil prices. Renewable energy is classified based on the energy resources; and wind is one of the attractive renewable energy resources. Offshore wind-power systems can generate higher power than onshore wind-power systems because of strong and stable winds. For this reason, the capacities of offshore windmills and power conditioning systems (PCSs) have been increasing. For the offshore windmills, the neutral point clamped (NPC) topology has been largely adopted because of the advantage that it can be implemented within a limited space [1,2]. In order to apply the NPC topology, gate drive units capable of stably driving large power are needed.

Insulated gate bipolar transistor (IGBT) is generally used for a medium-capacity-class PCS with a switching frequency of several tens of $\mathrm{kHz}$. For a high capacity, the power stack is designed using IGBTs connected in series or in parallel. The performance of the IGBTs is determined by the gate drive. The switching characteristics and waveform control are also closely related to this performance. A poorly designed gate driver may damage the IGBTs and the peripheral circuit. Therefore, the gate driver should be designed in order to achieve the correct output for reliable operation. For high-voltage and high-capacity PCSs, performance of the IGBT gate driver is a critical issue because of the high possibility of causing a safety problem such as a short-circuit failure.

$\dagger$ Corresponding Author: Dept. of Electrical and Computer Engineering, Sungkyunkwan University, Korea. (won@yurim.skku.ac.kr)

* Intelligent Mechatronics Research Center, Korea Electronics Technology Institute, Korea. ( \{kimjinhong, parkjs\}@keti.re.kr)

** Dept. of Energy Engineering, Kyungpook National University, Korea. (bggu@knu.ac.kr)

Received: January 29, 2016; Accepted: June 1, 2016
The IGBT gate drivers are classified into: those based on a passive driving method and those based on an active driving method. In the passive driving method, the IGBT gate drive circuits have fixed gate resistors. These resistors are selected to suppress the switching electromagnetic interference (EMI) to an acceptable level and limit the reverse-recovery current with minimal energy loss $[3,4]$. While meeting these requirements, the passive driving methods fail to achieve a good performance (such as short switching delay and high efficiency). The gate drivers using these methods have low complexity and are inexpensive. However, the passive driving methods have high switching losses. It is important to minimize these losses, particularly, the converter switching loss for a high power capacity. To overcome the switching delay and switching loss problems, various active driving approaches have been proposed in the past [5-13]. The methods in [510] have used multiple gate resistors to control the gate current during switching transients. These methods provide a simple and common way of adjusting the switching speed. The gate voltage control methods in [11-13], control the gate current using gate voltage. In these methods, the generation of reference voltage is more complex than in the case of switchable resistors. These active gate drive methods can be classified into open-loop control and closed-loop control methods. With open-loop control, it is difficult to obtain the switching timing or target voltage under all operating conditions. For this reason, the closed-loop control method is used to generate a precise gate signal.

This paper presents a gate drive unit with closed-loop control using the slope of the $d i / d t$ signal that can be obtained from the inductance between the kelvin-emitter and the power-emitter as shown in Fig. 3. In the proposed method, the turn-on sequence is determined by the $d i / d t$ feedback signal. This analog feedback signal is converted to a digital signal for suppressing the switching noise, and 


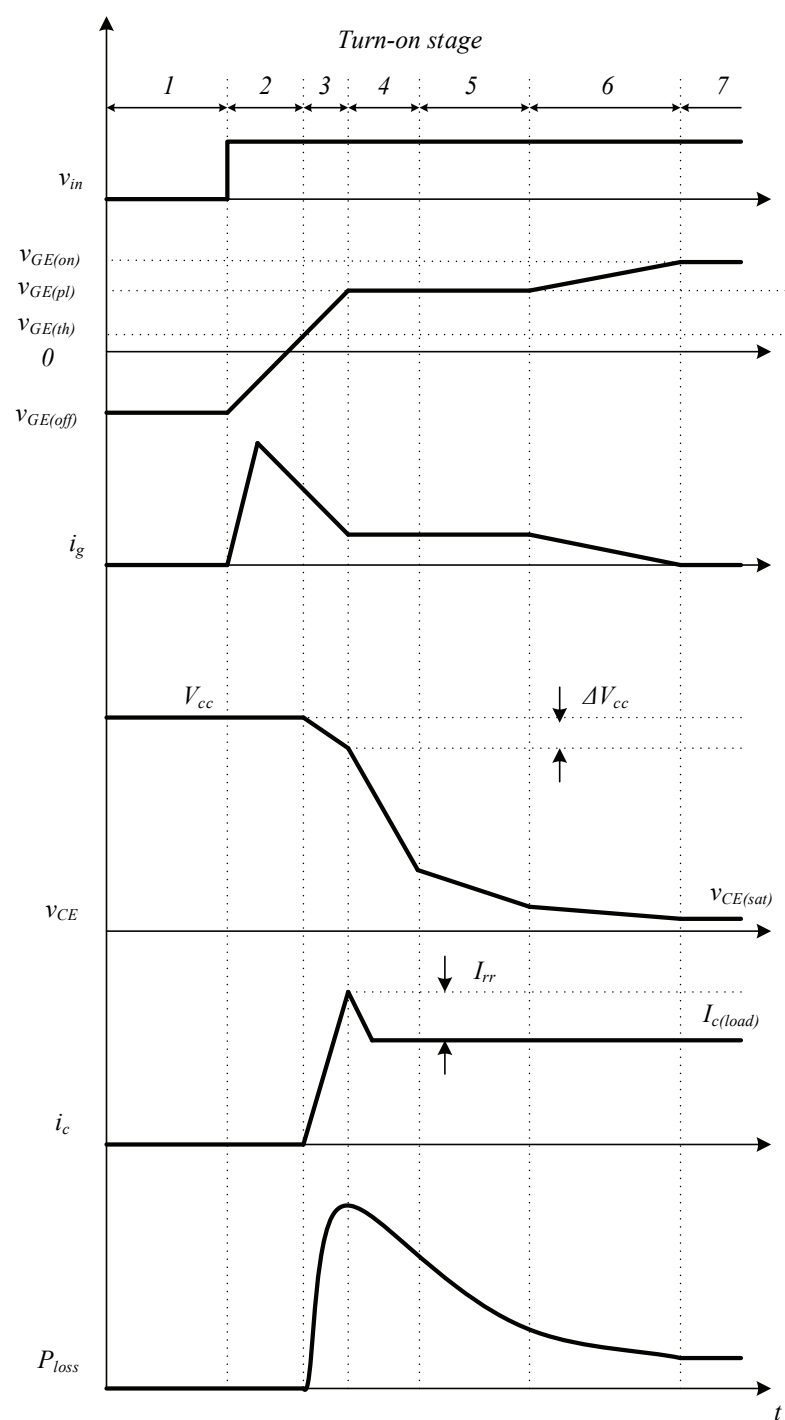

(a)

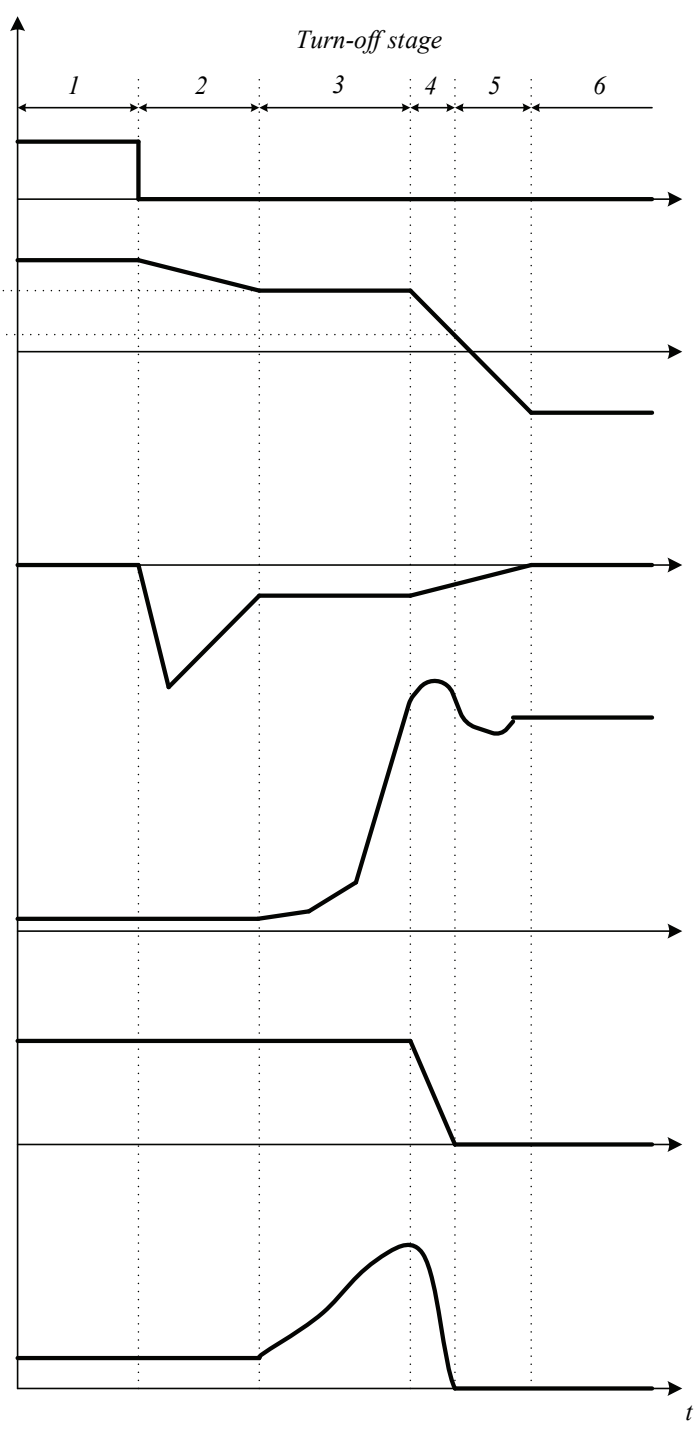

(b)

Fig. 1. Sketches of (a) turn-on and (b) turn-off waveforms of an IGBT

the digital closed-loop control based on multiple gate resistors is created. The proposed gate drive method is experimentally verified and the results are presented in this paper.

\section{IGBT Operation}

The structure of a bipolar junction transistor (BJT) ensures that it has both majority and minority carriers available during the conductive phase. Therefore, the internal voltage drop of a BJT, when it is turned on, is less than that of a MOSFET with a comparable current and blocking voltage. In contrast, a MOSFET can be turned on at the gate with less current than a BJT, which requires a relatively high current to be maintained at $\mathrm{ON}$ state. The combination of the gate behavior of a MOSFET with the conduction behavior of a BJT results in an IGBT [14].
Typical waveforms at turn-on and turn-off of an IGBT are sketched in Fig. 1. The turn-on stage can be distinguished over seven periods as shown in Fig. 1(a). The first stage is the OFF state, before the gate signal is applied. After the gate signal is applied, the gate current $\left(i_{g}\right)$ charges the input capacitance $\left(C_{G E}\right)$ and the gate-emitter voltage $\left(V_{G E}\right)$ rises to $V_{G E(t h)}$. This is the second stage. However, the IGBT still does not switch current until $V_{G E}$ exceeds $V_{G E(t h)}$. Depending on the gate resistor, $i_{g}$ is determined and the duration of the second stage is also determined. This stage thus represents the turn-on delay. During this phase, the delay time $\left(t_{d(o n)}\right)$ can be estimated by neglecting parasitic inductances as

$$
t_{d(o n)}=-R_{g} C_{i e s} \ln \left(\frac{V_{G E(o n)}-V_{G E(o f f)}}{V_{G E(o n)}-V_{G E(t h)}}\right),
$$

where $C_{i e s}\left(C_{i e s}=C_{G E}+C_{G C}\right)$ is the input capacitance of the 
IGBT [7, 8]. The delay time is determined by the gate resistance or gate current. In this stage, the collect current has not appeared and $V_{C E}$ is maintained at $V_{C C}$ level. The actual turn-on process starts after $V_{G E}$ has reached $V_{G E(t h)}$. At this third stage, $I_{c}$ begins to increase and $V_{C E}$ begins to decrease. Because of a reverse-recovery current, $I_{c}$ exceeds $I_{c \text { (load) }}$ and $V_{C E}$ does not reach zero. From the equivalent circuit shown in Fig. 3, the following relationships can be derived:

$$
\begin{gathered}
I_{c}(t)=g_{m}\left(v_{G E}-v_{G E(t h)}\right) \\
L_{E 1} \frac{d i_{c}}{d t}+v_{G E}+R_{g} C_{i e s} \frac{d v_{G E}}{d t}=v_{G E(o n)}
\end{gathered}
$$

$L_{G}, L_{C}, L_{E 1}$ and $L_{E 2}$ represent the interconnection and bond-wire inductances of the gate, collector, kelvin-emitter and power-emitter respectively. $C_{G E}, C_{G C}$ and $C_{C E}$ represent the parasitic capacitances between three terminals.

The collect-current rise slope can be described using (2) and (3) as
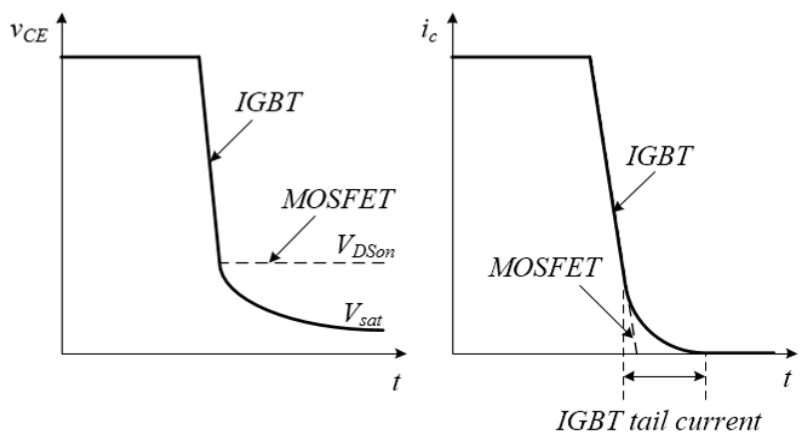

Fig. 2. Comparison of turn-on and turn-off operation of MOSFETs and IGBTs

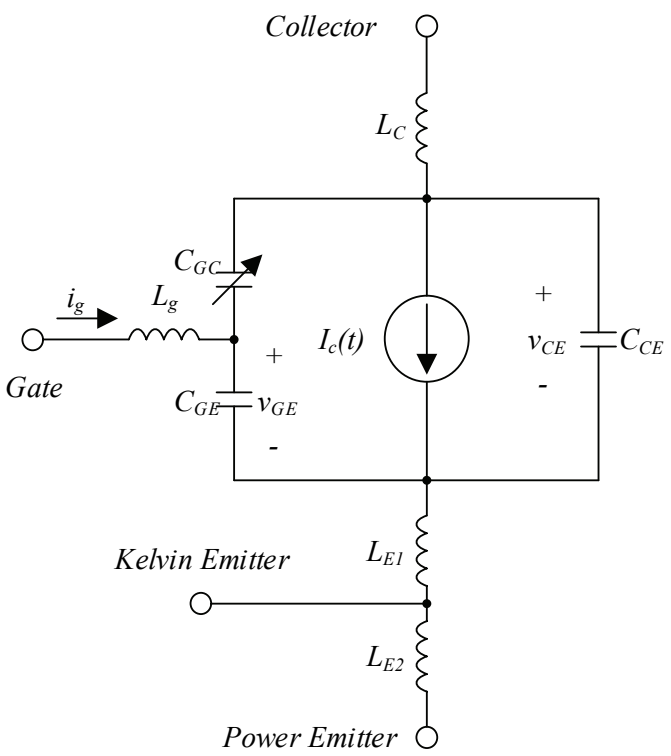

Fig. 3. IGBT model

$$
\frac{d i_{c}}{d t}=\frac{v_{G E(o n)}-v_{G E}}{L_{E 1}+\frac{R_{g} C_{i e s}}{g_{m}}}
$$

As shown in (4), the slope of current can be increased by selecting a smaller value for the gate resistor. The selection of the gate resistor is important to reduce the turn-on loss and the reverse-recovery current. At the fourth stage, $V_{G E}$ maintains the plateau voltage $V_{G E(p l)}$. When the freewheeling diode is turned off, $V_{C E}$ starts to drop rapidly. At the fifth stage, the miller capacitance $\left(C_{G C}\right)$ increases as the voltage decreases and is charged by $i_{g} ; V_{G E}$ still remains at the $V_{G E(p l)}$ level. At the beginning of the sixth stage, the IGBT is fully turned on. The charge conducted to $C_{G E}$ induces an increase in $V_{G E}$ up to the gate signal voltage $V_{G E(o n)}$. The gate current $\left(i_{g}\right)$ goes down to zero and $V_{C E}$ reaches $V_{C E(s a t) \text {. }}$ At the seventh stage, $i_{g}$ is zero and the turn-on state is completed. The turn-off stages are shown in Fig. 1(b). During the turn-off stages, the processes run in reverse direction. Fig. 1(b) shows the delay before the collectorvoltage rise, the overvoltage during the collector-current fall, and the tail phenomena at the end of the collectorcurrent fall occurring during the turn-off stages [3, 19-23].

\section{Gate Driving Methods}

\subsection{Passive gate driving method}

Fig. 4 shows a block diagram of a passive gate drive unit which is widely used and accepted in the industry. The passive gate drive is composed of a current amplifier to charge the input capacitances of the IGBTs, and turn-on/off gate resistors for limiting the gate current $[3,4]$. The gate voltages of the IGBTs are selected based on the output characteristics of the IGBTs.

This gate drive unit has the advantage of a relatively low cost. However, the gate current cannot be varied. The gate current should be determined considering the turn-on loss and the freewheeling-diode current. In order to reduce the turn-on time, the gate current should be increased. However, this leads to increasing the reverse-recovery current at the turn-on state and the surge voltage at the

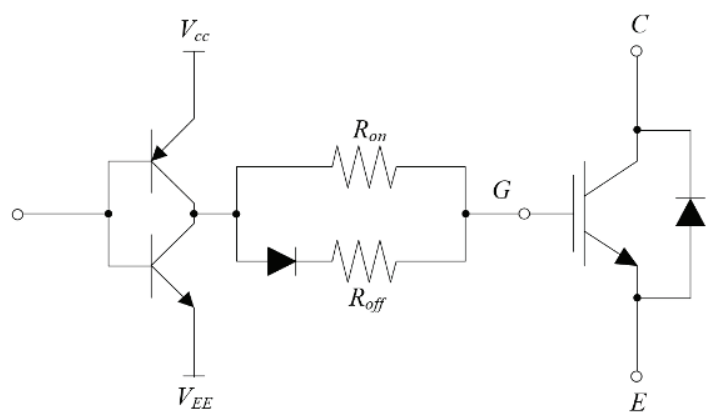

Fig. 4. Drive circuit for passive gate drive 


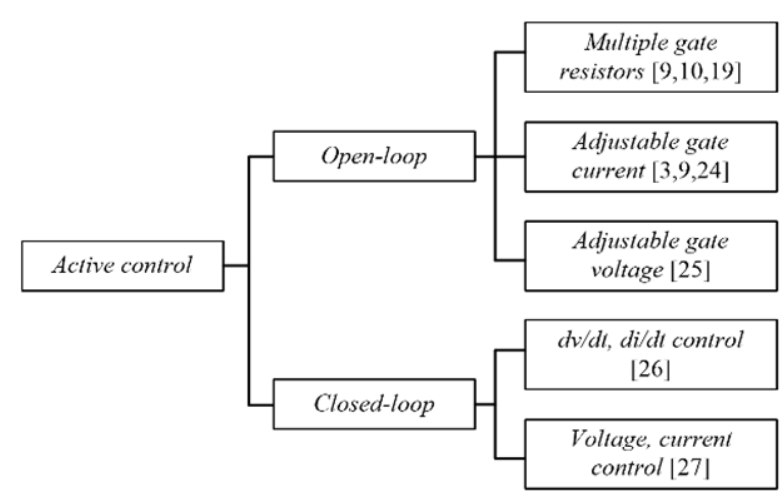

Fig. 5. Classification of gate driving method [28]

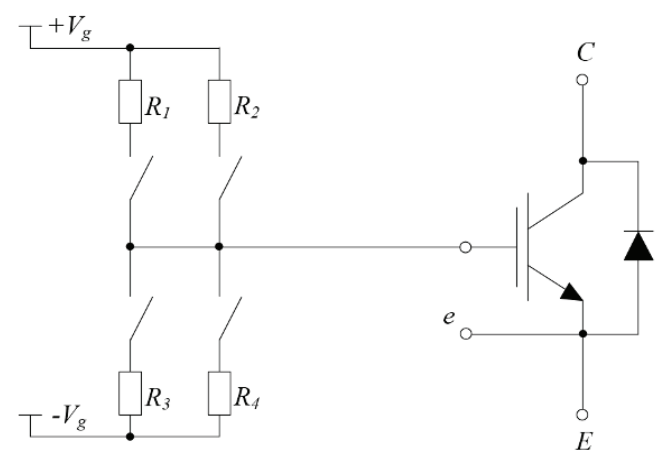

Fig. 6. Drive circuit with switchable gate resistors

turn-off state.

Stable gate driving is possible by using the passive gate driving method. Because the passive gate driving scheme suppresses the surge voltage due to the parasitic inductance components in the commutation loop of the converter system, the voltages and currents are within the Safety Operation Area (SOA). However, there could be an increase in the turn-on/off delay and switching losses [9, $14]$.

\subsection{Active gate driving method}

Fig. 5 classifies gate driving methods into open-loop and closed-loop types. The resistive push-pull gate driver, which consists of a switchable voltage source and turnon/off gate resistors, is a very simple and, thus, a widely used. The use of low-ohmic gate resistors leads to high absolute values of the gate current, which results in fast switching transients [28, 29].

Fig. 7 shows a block diagram of the gate driving scheme based on controlling the gate voltage or current. The gate voltage or current is controlled using a predetermined turn-on/off pattern such as a look-up table. The output profile is generated after analysis of the IGBT switching characteristics in each section [11, 14-16]. By this method, the switching losses can be reduced because the turn-on/off delay, $d i / d t$ and $d v / d t$ at the constant output current region can be reduced. However, the switching characteristics

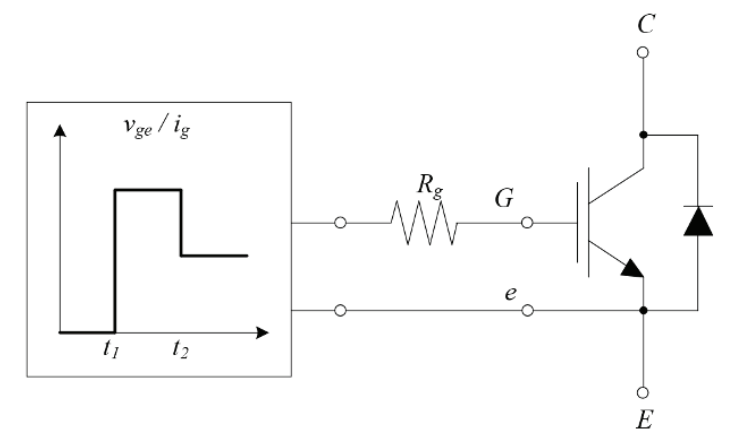

Fig. 7. Drive circuit for open-loop active gate drive

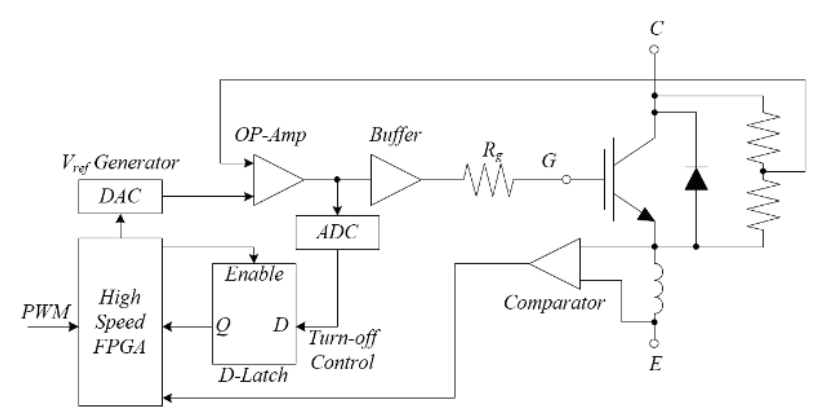

Fig. 8. Drive circuit for closed-loop active gate drive

may be changed by the collector current of the IGBTs or by the junction temperature. Therefore, the profile should be designed considering these adverse conditions. However, by this method, it is difficult to improve the switching efficiency in the entire operating area.

Fig. 8 shows a block diagram of the closed-loop active gate driving method in which $d i / d t$ and $d v / d t$ are controlled by digital and analog controllers $[13,17,18]$. The digital controller is operated by the feedback voltage of the emitter parasitic inductance, and the gate voltage is controlled during switching transients by the analog controller. However, configuring precise and real time control is difficult due to the conversion time and resolution of a digital-to-analog converter (DAC) and an analog-to-digital converter (ADC). For these reasons, the gate driving method, which operates at the next switching state using the information analyzed from the previous switching state, has been studied [18]. However, it is difficult to apply this method to a sudden load change or an unexpected accident.

\section{Proposed Active Gate Driving Method}

Fig. 9 shows the proposed gate drive circuit with multiple resistors. A single positive voltage is used as a voltage source and the gate voltage is generated by the $\mathrm{P} / \mathrm{N}$-type MOSFETs. The upper and lower switches are operated complementarily. Using a combination of eight MOSFETs, the gate-emitter voltage $\left(V_{G E}\right)$ is determined. 6 switches $\left(\mathrm{S}_{1}-\mathrm{S}_{6}\right)$ determine the gate terminal-voltage and 
two switches $\left(\mathrm{S}_{7}, \mathrm{~S}_{8}\right)$ determine the emitter terminalvoltage. Positive or negative voltage is generated using a combination of these two terminal voltages. The gate resistance can be set by a combination of gate resistors $\left(\mathrm{R}_{1^{-}}\right.$ $\mathrm{R}_{8}$ ) which are connected to the MOSFETs. During turn-on or turn-off sequences, the gate resistance can be changed using combinations of these gate resistors. The proposed gate drive circuit can generate seven kinds of resistances during the turn-on and turn-off sequences.

Fig. 10 shows the turn-on and turn-off switching waveforms when using different gate-resistance values. As shown in (4), when we select a small value for gate resistance, the slope of current is large and the time taken to reach the maximum load current is reduced accordingly. However, the diode reverse-recovery current becomes large. Therefore, in this case, the IGBT circuit should be designed considering the diode reverse-recovery current. Further, if a large gate resistance is selected, the slope of

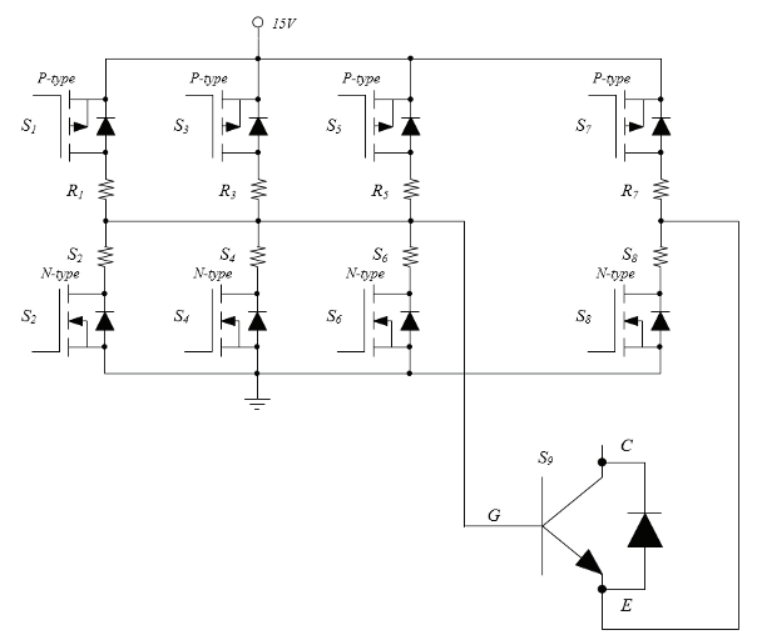

Fig. 9. Proposed gate drive circuit with multiple resistors

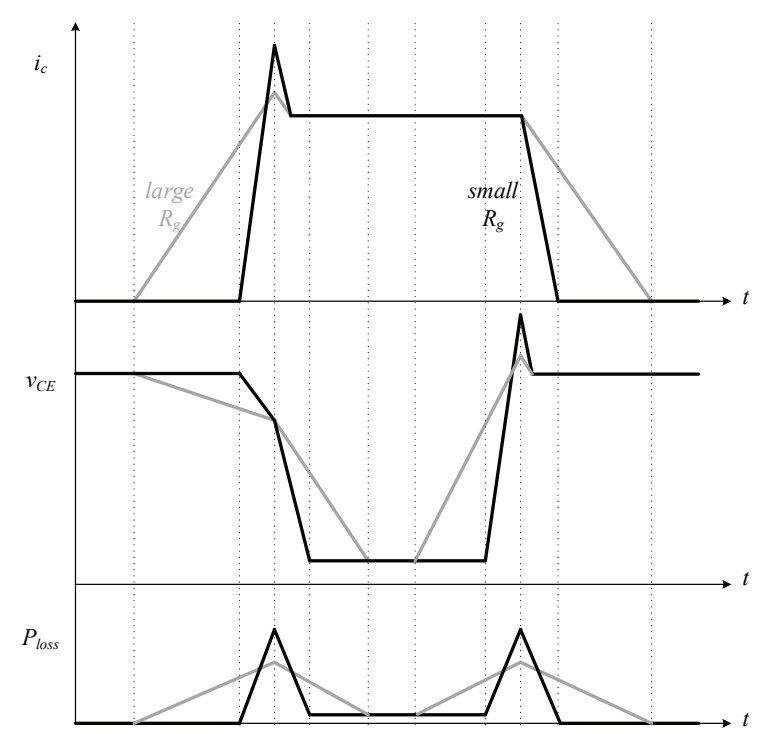

Fig. 10. Comparison of switching waveforms when using different gate-resistance values current is small and the diode reverse-recovery current is small accordingly. In this case, the switching time is longer and the switching loss larger.

The voltage drop between the kelvin-emitter and the power-emitter parasitic inductance $L_{E 2}$, of the IGBT, is given by the following equation:

$$
V_{L E}=-L_{E 2} \frac{d i_{c}}{d t}
$$

As shown in (5), the slope of the current $-d i_{c} / d t$ can be obtained by the voltage drop between the kelvin-emitter and the power-emitter. This voltage drop is used as a source of feedback for the proposed active gate driving method during the turn-on sequence. For the turn-off operation, the $d v / d t$, which is obtained from the external diode and resistor, is used as the feedback source.

By controlling the gate-resistor value, active gate driving control can be achieved. Increasing the value of the gate resistor causes an increased switching delay, and a softer switching transient. A lower $d i_{c} / d t$, which occurs with a larger gate resistance, increases the switching loss. Therefore, selecting a proper resistor value is important for improving the switching loss at each sector of the switching transient.

Fig. 11 shows the block diagram of the proposed active gate driving method. The turn-on sequence of the proposed method are divided into three regions. During each of three intervals, the gate resistance is adjusted to a different value. In the turn-on sequence, the $d i_{c} / d t$ signal is used to determine the switching region. In Fig. 12, the switching waveforms of the proposed method are shown. In region I of the turn-on period, we set a small value for gate resistance in order to rapidly increase the gate-emitter voltage $\left(v_{G E}\right)$ to the threshold voltage. In region II of turnon period, $v_{G E}$ has increased beyond the threshold voltage.

In this period, a larger value for resistance is selected in order to reduce the spike voltage. The $d i_{c} / d t$ signal is continuously detected and a digital signal is generated when the value of $d i_{c} / d t$ is negative. If the collect current reaches the maximum, the digital output of $d i_{c} / d t$ becomes

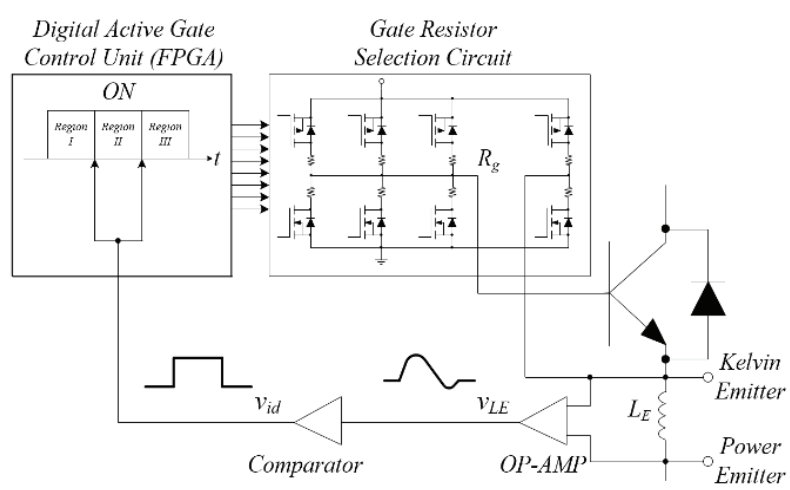

Fig. 11. Block diagram of the proposed active gate driving method 


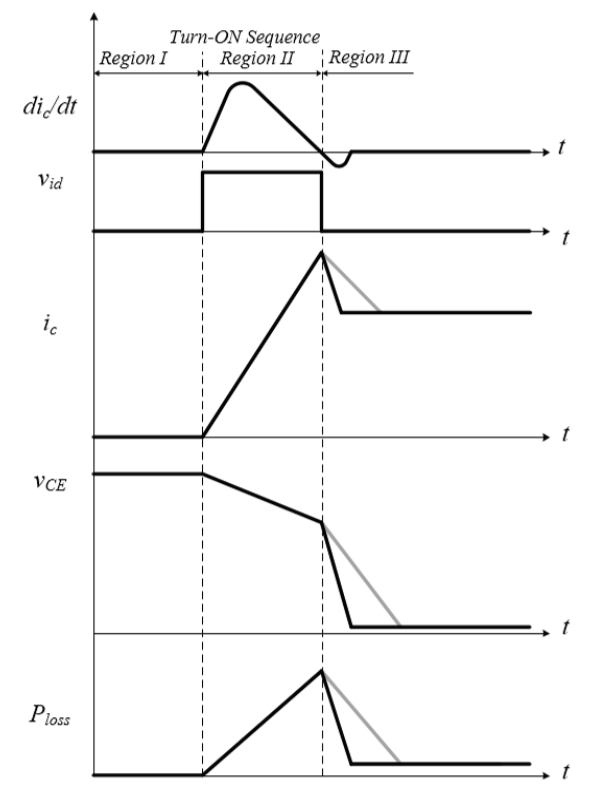

Fig. 12. Switching waveforms of the proposed gate drive method zero. From this moment, region III starts. In region III of the turn-on period, a small resistance value is selected to rapidly decrease the collector-emitter voltage $\left(v_{C E}\right)$ to the saturation voltage.

As illustrated in Fig. 13, the operation mode of the proposed active gate driver is divided into five sectors. Before the gate signal injected, $S_{2}, S_{4}, S_{6}$, and $S_{7}$ switches are turned on. And negative gate signal can be generated. In order to prevent a short circuit, dead time is injected as shown in Fig. 13 (b) before the turn-on operation. In the sector shown in Fig. 13 (c), maximum gate current is injected for compensating turn-on delay. For generating the maximum gate current, minimum gate resistance is applied. Before the collector current is generated, this operation is kept up. In the sector in Fig. 13 (d), for reducing the diode reverse recovery current, small gate current as the conventional method is generated. This operation mode is kept up until the digital signal is changed by $d i_{c} / d t$. After changing $v_{i d}$, the maximum gate current is injected to reduce the time to reach the steady state of collector-

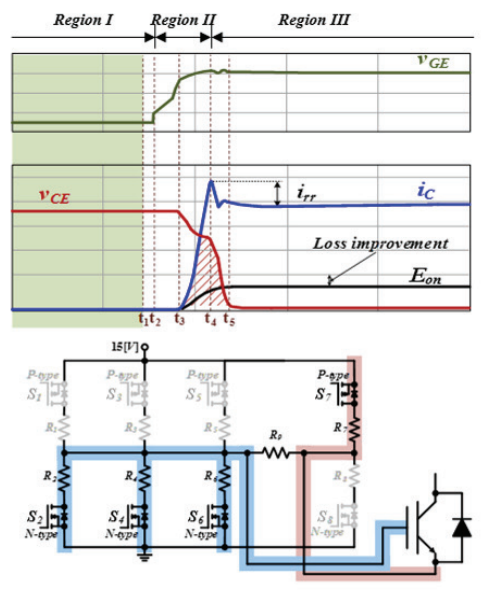

(a)
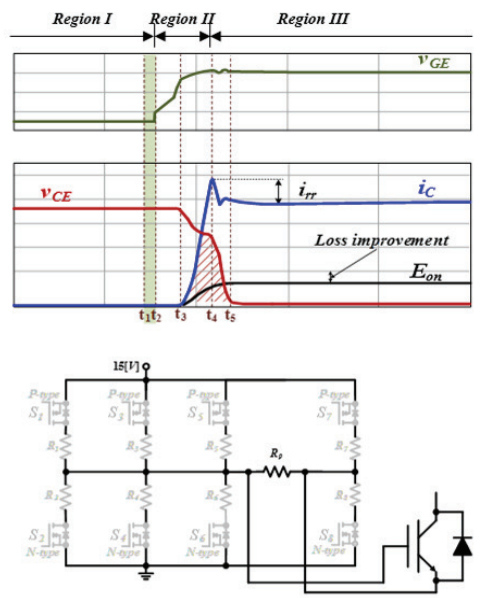

(b)
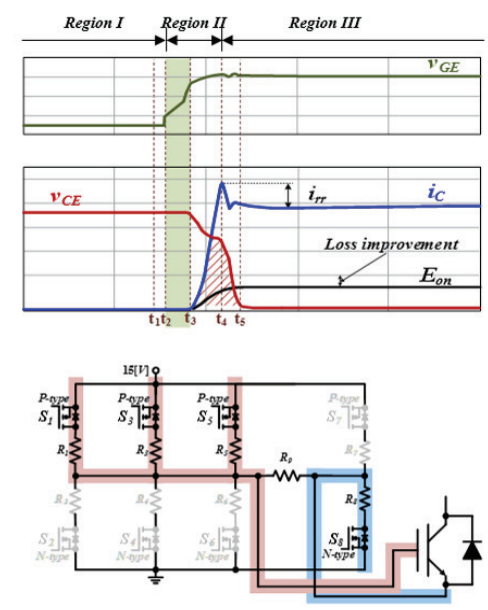

(c)
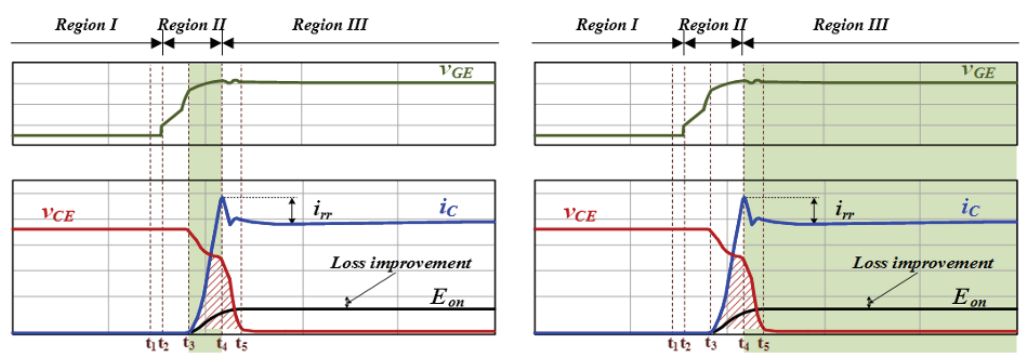

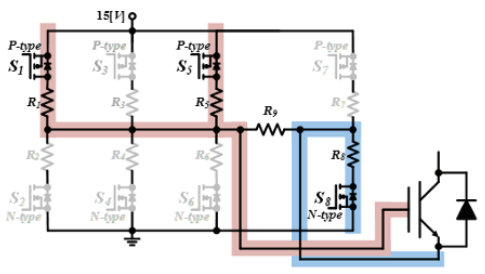

(d)

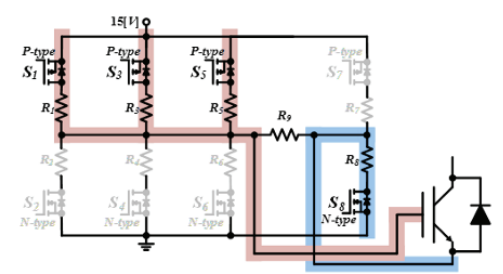

(e)

Fig. 13. Operation mode and gate current pass for proposed method at (a) sector from turn-off to $t_{1}$, (b) sector from $t_{1}$ to $t_{2}$, (c) sector from $t_{2}$ to $t_{3}$, (d) sector from $t_{3}$ to $t_{4}$, and (e) sector from $t_{4}$ to turn-on. 


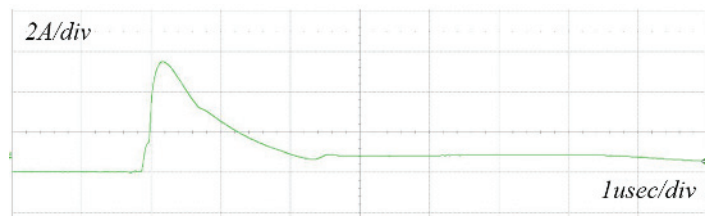

(a)

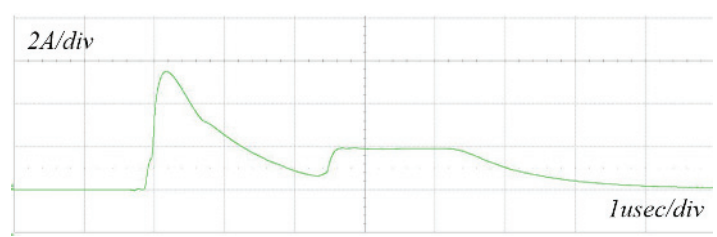

(b)

Fig. 14. Gate current waveforms of (a) conventional method and (b) proposed method.

emitter voltage and collector current as shown in Fig. 13 (e). By this operation, the $d v / d t$ of collector-emitter voltage is increased. And the intersection area of collector-emitter voltage $v_{C E}$ and gate current $i_{c}$ is decreased. Finally, the switching loss is decreased. Fig. 14 shows the gate current waveforms of conventional method and proposed method. At the initial turn-on section, both waveforms are similar. However, at the region III, gate current using proposed method is quite large. As mentioned above, due to the large gate current, the fast switching operation is made.

\section{Experimental results}

Table 1 shows the specifications of MOSFETs which is applied for IGBT switching. Considering MOSFETs switching speed, the dead time is applied for the reliable switching operation as $0.1 \mathrm{usec}$.

Fig. 15 shows a hardware prototype of the proposed active gate drive, and a test bench for validating the proposed active gate driving method. The proposed method is implemented on a Complex Programmable Logic Device (CPLD). The test bench consists of a 1-arm IGBT switch with the gate drive circuit. A 4.5-kV, 1,200-A IGBT (Mitsubishi CM1200HG-90R) is used. Fig. 16 shows a test configuration and the operation of the gate drive. Two pulse tests have been performed.

\subsection{Conventional passive gate drive method}

In order to analyze the switching characteristics of the turn-on stage, the passive gate drive test was performed by setting the gate resistance and the collector current as shown in Table 2.

Fig. 17 shows the waveforms of the pulse test in the conventional passive gate drive. As shown in Fig. 17, the slope of $d i_{c} / d t$ is determined by the gate resistance. When using the same resistance, the $d i_{c} d d t$ has the same slope
Table 1. Specifications of MOSFETs

\begin{tabular}{c|c|c}
\hline Type & N-type & P-type \\
\hline Turn-on delay time & $8.1 \mathrm{~ns}$ & $13 \mathrm{~ns}$ \\
\hline Rise time & $8.9 \mathrm{~ns}$ & $13 \mathrm{~ns}$ \\
\hline Turn-off delay time & $26 \mathrm{~ns}$ & $34 \mathrm{~ns}$ \\
\hline Fall time & $17 \mathrm{~ns}$ & $32 \mathrm{~ns}$ \\
\hline
\end{tabular}

Table 2. Switching test cases

\begin{tabular}{c|c|c}
\hline & Gate resistance & Collector current \\
\hline Case 1-1 & & $500 \mathrm{~A}$ \\
Case 1-2 & $2.0 \Omega$ & $800 \mathrm{~A}$ \\
Case 1-3 & & $1,100 \mathrm{~A}$ \\
\hline Case 2-1 & $4.1 \Omega$ & $500 \mathrm{~A}$ \\
Case 2-2 & $800 \mathrm{~A}$ \\
Case 2-3 & & $1,100 \mathrm{~A}$ \\
\hline Case 3-1 & \multirow{2}{*}{$6.0 \Omega$} & $500 \mathrm{~A}$ \\
Case 3-2 & & $800 \mathrm{~A}$ \\
Case 3-3 & & $1,100 \mathrm{~A}$ \\
\hline
\end{tabular}

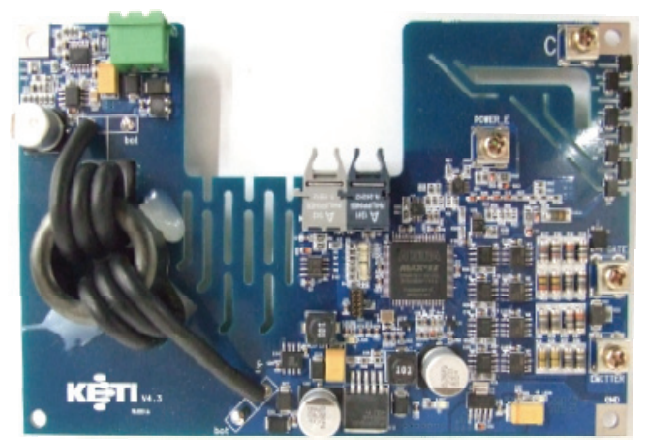

(a)
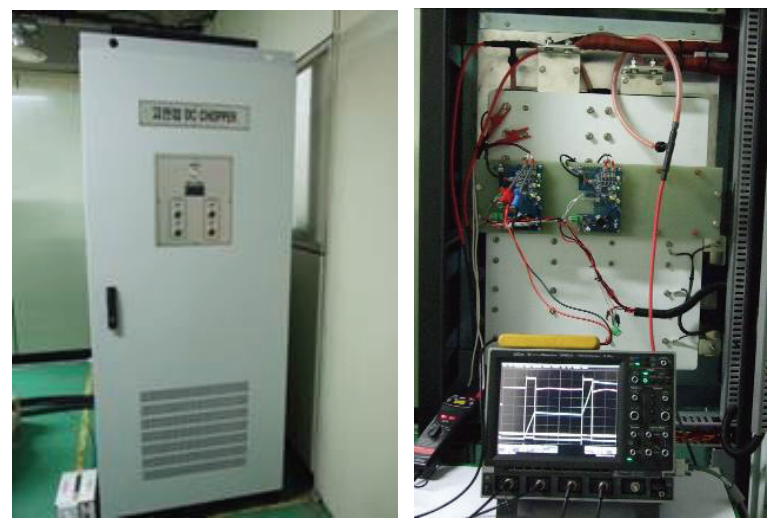

(b)

Fig. 15. Picture of (a) gate drive board and (b) test bench

irrespective of the value of current. The slope of $d v / d t$ is also affected by the gate resistance. Fig. 17(d) shows a comparison of the switching losses. All switching losses have the same slope and the loss is determined by the current.

Fig. 18 shows the waveforms when the gate resistance is varied with the current remaining the same. A small gate resistance generates a large gate current $\left(i_{g}\right)$. This gate current charges the input capacitance $\left(C_{G E}\right)$, and $V_{G E}$ 
rapidly rises to $V_{G E(t h)}$.

As a result of these operations, the switching time is

Table 3. Switching energy loss comparison

\begin{tabular}{c|c|c|c}
\hline & $500 \mathrm{~A}$ & $800 \mathrm{~A}$ & $1,100 \mathrm{~A}$ \\
\hline $\mathrm{E}_{\text {on.passive }}(\mathrm{J})$ & 1.58 & 2.65 & 4.07 \\
\hline $\mathrm{E}_{\text {on.active }}(\mathrm{J})$ & 1.43 & 2.32 & 2.83 \\
\hline Loss reduction (\%) & 9.5 & 12.5 & 15.1 \\
\hline
\end{tabular}

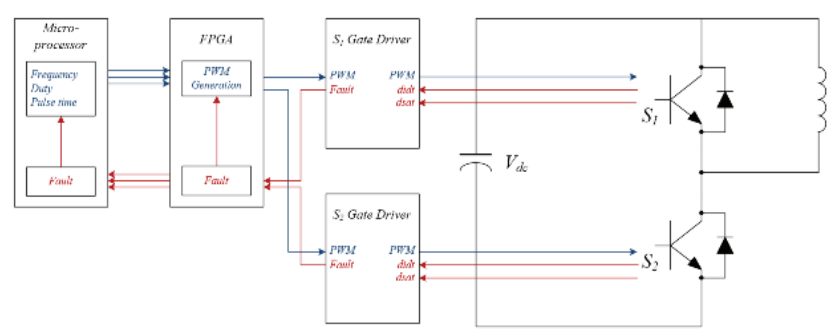

(a)

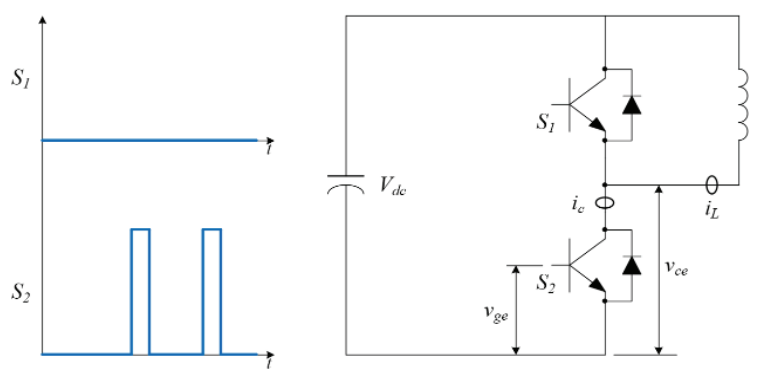

(b)

Fig. 16. (a) test configuration for gate drive and (b) operation of gate drive circuit

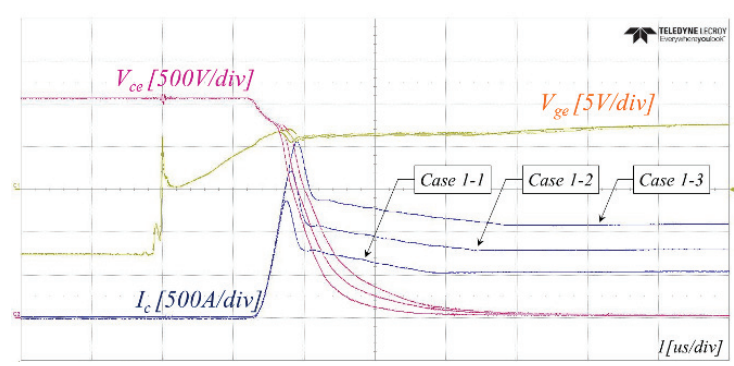

(a)

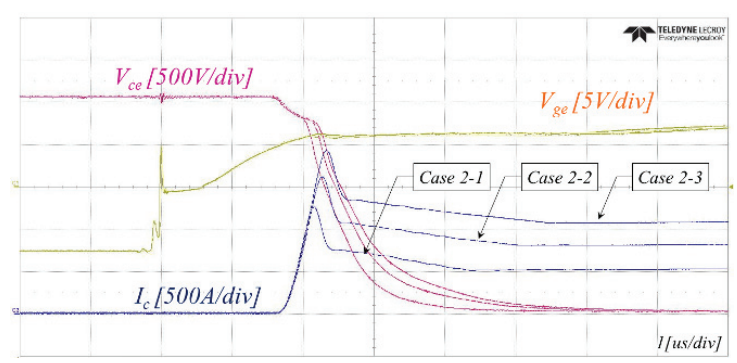

(b) shortened. However, the diode reverse-recovery current becomes large as shown in Fig. 18. Therefore, it is important to design the gate resistance properly in the passive gate drive.

\subsection{Proposed active gate drive method}

The trade-off in the turn-on characteristics of the switching time, the peak reverse-recovery current, and the power dissipation is shown in Fig. 17 and Fig. 18. As the gate resistance increases, the switching time and the dissipated energy also increases, while the reverserecovery current decreases. For the proposed active gate drive, different gate resistors are used in each region, whose values are determined by the $d i_{c} / d t$ signal. Before the $d i_{c} / d t$ signal becomes negative, the operation of the proposed method is similar to that of the conventional method as shown in Fig. 19. It operates such that the resistance is set to be smaller when the $d i_{c} / d t$ signal becomes negative.

Fig. 19 shows a comparison of the conventional passive gate drive and the proposed active gate drive.

The switching time of the proposed method is shorter than that of the conventional method and $V_{c c}$ of the proposed method reaches zero more quickly even though the peak reverse- recovery is the same. Table 3 shows the comparison of the switching energy losses. The switching energy loss of the proposed method decreases under all load conditions. The improved performance of the proposed active gate drive holds good over a wide range of current levels.

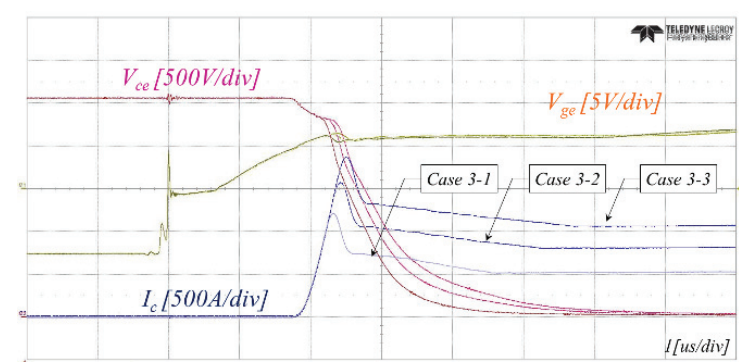

(c)

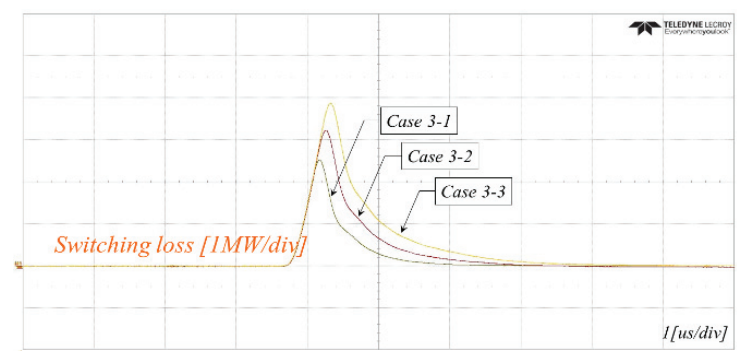

(d)

Fig. 17. Switching characteristics when collector current increases at (a) $2 \Omega$, (b) $4.1 \Omega$, (c) $6 \Omega$, and (d) switching loss comparison at $6 \Omega$. 
Turn-on Loss Reduction for High Voltage Power Stack Using Active Gate Driving Method

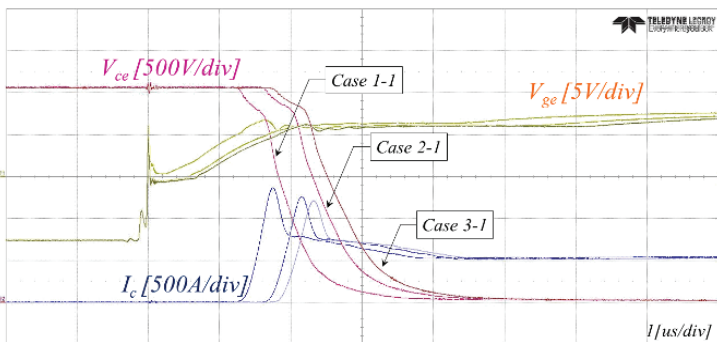

(a)

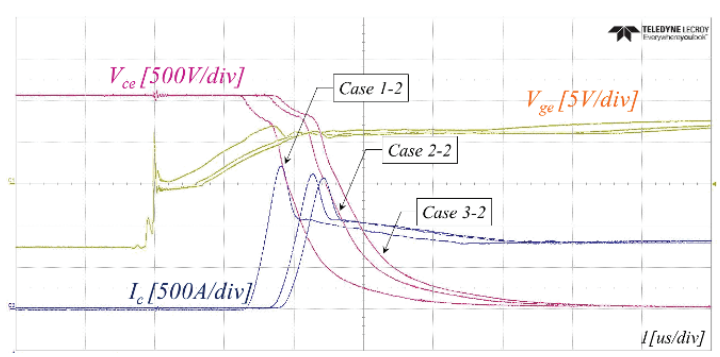

(b)

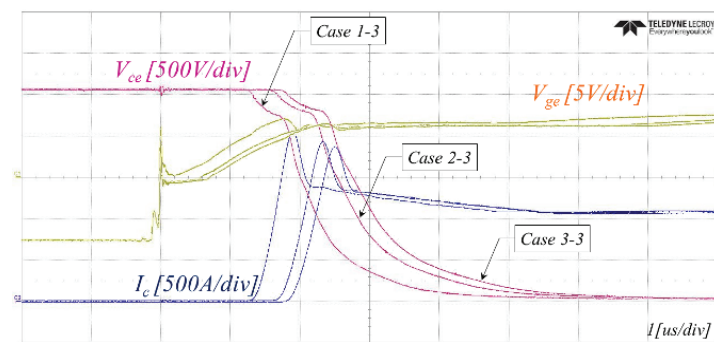

(c)

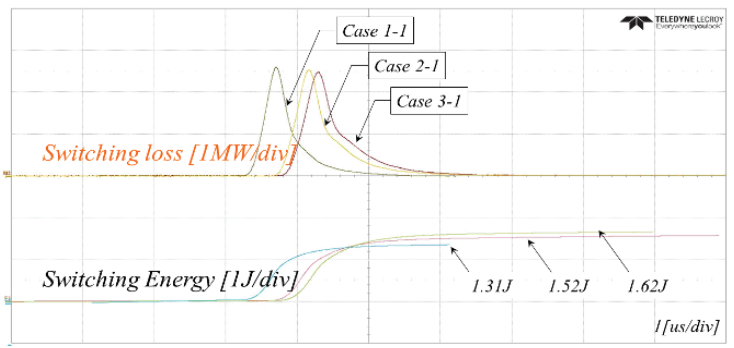

(d)

Fig. 18. Switching characteristics when gate resistance increases at (a) 500A, (b) 800A, (c) 1,100A, and (d) switching loss comparison at 500A.

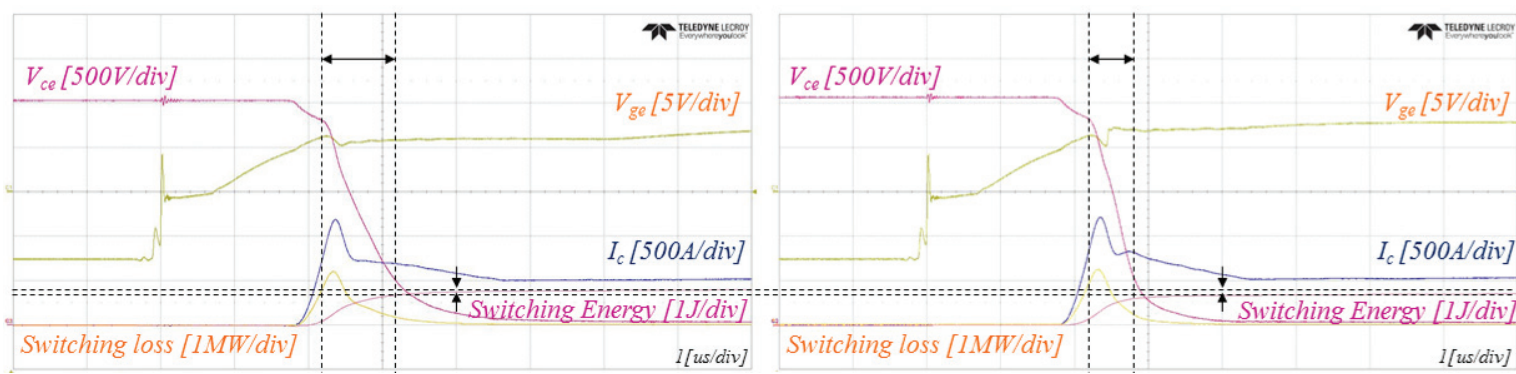

(a)

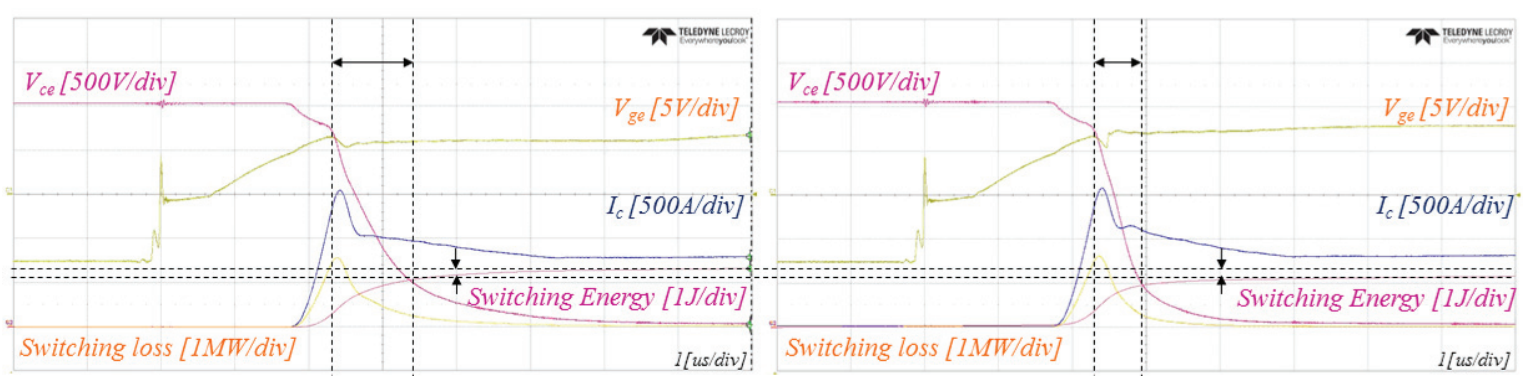

(b)

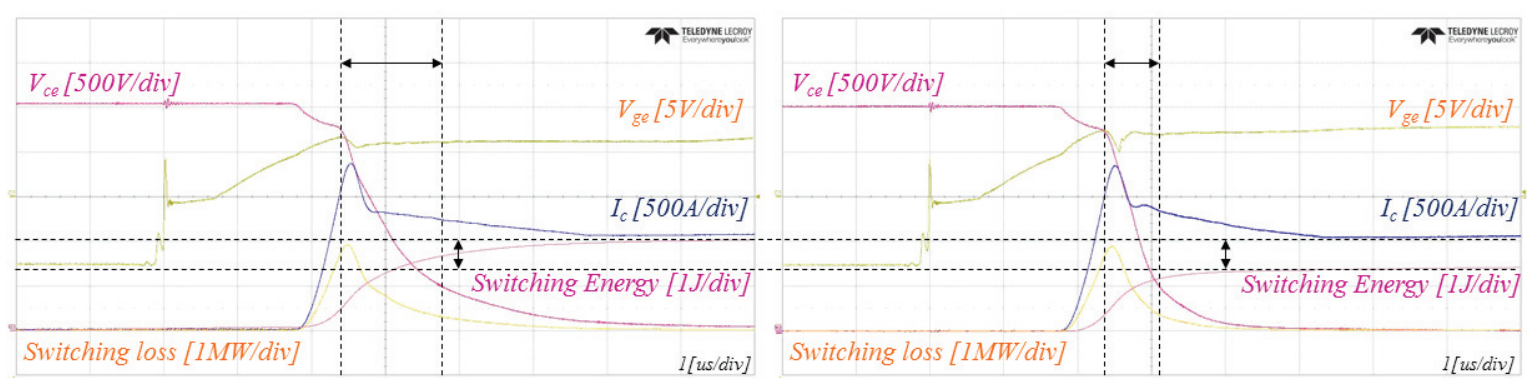

(c)

Fig. 19. Comparison of conventional gate drive and proposed method at (a) 500A, (b) 800A, and (c) 1,100A 


\section{Conclusion}

This paper presented an IGBT gate drive method for a medium-capacity-class PCS. The turn-on energy loss and switching time for a conventional method and the proposed method was analyzed, compared and presented in the paper. In the proposed active gate drive, the $d i_{c} / d t$ signal was used to distinguish the switching region and a different gate resistor was selected in each region. The $d i_{c} / d t$ signal generator and the configuration of the feedback loop are simple. An experimental set up was created, and several tests were carried out for verifying the proposed method and comparing it with the conventional method. The proposed method provided improved switching energy loss and switching time under a wide range of load conditions.

\section{Acknowledgments}

This work was supported by the Korea Institute of Energy Technology Evaluation and Planning(KETEP) and the Ministry of Trade, Industry \& Energy(MOTIE) of the Republic of Korea (No. 20162010103830).

\section{References}

[1] Wu B, Lang Y, Zargari N, and Kouro S, Power conversion and control of wind power systems, WileyIEEE Press, 2011.

[2] Rodriguez J, Bernet S, Wu B, Pontt JO, and Kouro S, "Multilevel voltage source converter topologies for industrial medium-voltage drives," IEEE Trans. Ind Electron., vol. 54, no. 6, pp. 2930-2945.

[3] V. John, B. S. Suh and T. A. Lipo, "High-performance active gate drive for high-power IGBT's," IEEE Trans. Ind. Appl., vol. 35, no. 5, pp. 1108-1117, Sept./Oct., 1999.

[4] L. Heinemann, J. Mast, G. Scheible, T. Heinzel, and T. Zuellig, "Power supply for very high insulation requirements in IGBT gate-drives," in Proceedings of IEEE Industry Applications Conference Soc. Annual Meeting, vol. 2, pp. 1562-1566, 1998.

[5] H. G. Eckel and L. Sack, "Optimization of the turnoff performance of IGBT at overcurrent and shortcircuit current," in Proceedings of EPE'93, pp. 317$322,1993$.

[6] Hemmer R., "Intelligent IGBT drivers with exceptional driving and protection features," in Proceeding of EPE'09, pp.1-4, 2009.

[7] Kieinichen P., Alvarez R., Buschendorf M. and Bernet S., "Turn-on loss reduction of $6.5 \mathrm{kV} / 500 \mathrm{~A}$ tench/field-stop IGBTs using a simple GDU," in Proceeding of IEEE ECCE 2013, pp. 3671-3678, 2013.

[8] Zhiqiang Wang, Xiaojie Shi, Yang Xue, Tolbert L.M., and Blalock B.J., "A gate drive circuit of high power IGBTs for improved turn-on characteristics under hard switching conditions," in proceeding of IEEE COMPEL 2012, pp.1-7, 2012.

[9] C. Licitra, S. Musumeci, A. Raciti, A. U. Galluzzo, R. Letor and M. Melito, "A new driving circuit for IGBT devices," IEEE Trans. Power Electronics, vol. 10, no. 3, pp. 373-378, May 1995.

[10] S. Takizawa, S. Igarashi and K. Kuroki, "A new di/dt control gate drive circuit for IGBTs to reduce EMI noise and switching losses," in Proceedings of IEEE PESC, vol. 2, pp. 1443-1449, 1998.

[11] Grbovic P.J. "An IGBT gate driver for feed-forward control of turn-on losses and reverse recovery current," IEEE Trans. Power Electronics, pp. 643-652, 2008.

[12] N. Idir, R. Bausiere and J. J. Franchaud, "Active gate voltage control of turn-on di/dt and turn-off $\mathrm{dv} / \mathrm{dt}$ in insulated gate transistors," IEEE Trans. Power Electronics, vol. 21, no. 4, pp. 849-855, Jul 2006.

[13] Y. Lobsiger and J. W. Kolar, "Closed-loop IGBT gate drive featuring highly dynamic di/dt and dv/dt control," in Proc. of IEEE ECCE, pp. 4754-4761, 2012.

[14] X.S. Yao, J. Feinberg, R. Logan, and L. Maleki, "Limitations on peak pulse power, pulse width, and coding mask misalignment in a fiber-optic codedivision multiple-access system", Journal of Lightwave Technology, vol. 11, no. 5, pp. 836-646, Jun 1993.

[15] Kiyoshi Yoshikawa et al., "Strongly Localized Potential Profile Measurements through Stark effects in the central core region of an inertial Electrostatic Fusion Device", Fusion Technology, vol. 39, no. 3, pp. 1193 〜 1201, 2001.

[16] A. Welleman and W. Fleischmann, "High Current, High di/dt Semiconductor Devices for Single and Repetitive Pulse Applications", in Proceedings of PPC-2003, Dallas TX, June 2003.

[17] Y. Wang, P. R. Palmer, T. C. Lim, S. J. Finney, and A. T. Bryant, "Realtime optimization of IGBT/diode cell switching under active voltage control," in Proceeding of the 41st IEEE Industry Applications Society Annual Meeting (IAS), vol. 5, Tampa, FL, USA, pp. 22622268, 2006.

[18] L. Dang, H. Kuhn, and A. Mertens, "Digital adaptive driving strategies for high-voltage IGBTs," in Proceeding of the IEEE Energy Conversion Congress and Exposition (ECCE), Phoenix, AZ, USA, pp. 2993-2999, 2011

[19] Andres Volke and Michael Hornkamp, IGBT Modules: Technologies, Driver and Application, Infineon, 2011

[20] Vinod Kumar Khanna, IGBT Theory and Design, Wiley, 2003.

[21] R. Chokhawala, J. Catt, and B. Pelly, "Gate drive considerations for IGBT Modules," in Proceedings of IEEE-IAS, pp.1186-1195, 1992.

[22] N. Mohan, T. M. Undeland, and W. P. Robbins, Power Electronics : Converters, Applications and 
Design, Wiley, 1995

[23] V. Venketashan, M. Eshaghi, R. Borras, and S. Deuty, "IGBT turn-off characteristics explained through measurements and device simulation," in Proceedings of IEEE APEC'97, pp. 175-178, 1997.

[24] A. Consoli, S. Musumeci, G. Oriti, and A. Testa, “An innovative EMI reduction design technique in power converters," IEEE Trans. Electromagn. Compat., vol. 38, no. 4, pp. 567-575, Nov. 1996.

[25] N. Idir, R. Bausiere, and J. J. Franchaud, “Active gate voltage control of turn-on $\mathrm{di} / \mathrm{dt}$ and turn-off $\mathrm{dv} / \mathrm{dt}$ in insulated gate transistors," IEEE Trans. Power Electron., vol. 21, no. 4, pp. 849-855, Jul.2006.

[26] L. Chen, and F. Z. Peng, "Closed-loop gate drive for high power IGBTs," in Proc. 24th IEEE Appl. Power Electron. Conf. Expo., Washington, DC, USA, Feb. 2009, pp. 1331-1337.

[27] Y.Wang, P. R. Palmer, T.C. Lim, S. J. Finney, andA. T.Bryant, "Real-time optimization of IGBT/diode cell switching under active voltage control," in Proc. 41st IEEE Ind. Appl. Soc. Annu. Meeting, Tampa, FL, USA, Oct. 2006, vol. 5, pp. 2262-2268.

[28] Y. Lobsiger and J. W. Kolar, "Closed-loop di/dt and dv/dt IGBT gate driver," IEEE trans. Power Electronics, vol. 30, No. 6, pp. 3402-3417, 2015.

[29] H. Kuhn, T. K"oneke, and A. Mertens, "Considerations for a digital gate unit in high power applications," in Proc. 39th IEEE Power Electron. Spec. Conf., Rhodes, Greece, Jun. 2008, pp. 2784-2790.

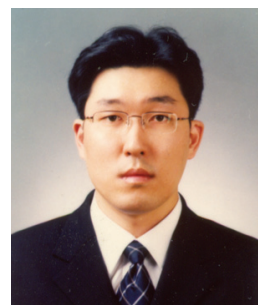

Jin-Hong Kim He was born in Korea, in 1976. He received the B.S., M.S., and Ph.D. degree in electrical engineering from Sungkyunkwan University, Suwon, Korea, in 2000, 2003, 2016, respectively. From 2003 to 2010, he was a researcher in LS industrial Systems, Korea. Since 2010, he has been a Senior Research Engineer at KETI (Korea Electronics Technology Institute), Bucheon, Korea. His research interests are AC motor control, PWM converter/inverter system, and electric energy conversions.

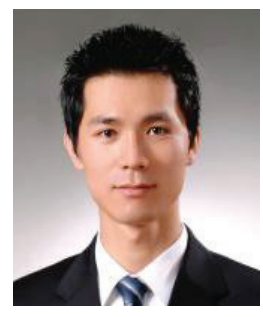

Joon Sung Park He was born in Seoul, Korea, in 1978. He received B. S. degree in Electrical and Electronic Engineering from Hongik University, Seoul, Korea, in 2005, and his M.S. degree in Electrical and Electronic Engineering from Pohang University of Science and Technology (POSTECH), Pohang, Korea, in 2007. From 2007 to 2009, he was with LG electronics, Seoul, Korea. Since 2009, he has been a
Senior Research Engineer at KETI (Korea Electronics Technology Institute), Bucheon, Korea. Since 2015, he has been pursuing the Ph.D. degree at the Department of Electrical Engineering, Hanyang University, Seoul, Korea. And he has also been an adjunct professor at Hanyang University, Seoul, Korea, since 2016. His current research interests include AC motor control, PWM converter, inverter system, and wave energy converter.

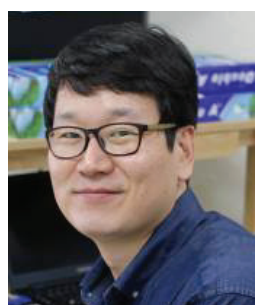

Bon-Gwan Gu received the B.S. degree from Kyungpook National University, Daegu, in 1998, and the M.S. and Ph.D. degrees from POSTECH, Pohang, Korea, in 2000 and 2005, respectively, all in electrical engineering. From 2005 to 2007, he was with LG electronics, Seoul, Korea. From 2008 to 2014, he worked as a managerial researcher at Korea Electronics Technology Institute, Bucheon, Korea. In 2014, he joined Kyungpook National University as an assistant professor in the School of Energy Engineering. His research interests are AC motor control, DC-DC converters, and PWM converter/inverter system.

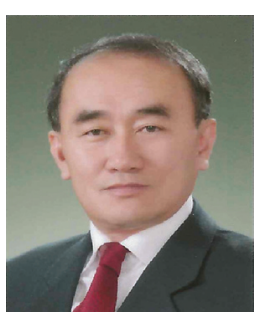

Chung-Yuen Won was born in Korea in 1955. He received the B.S. degree from Sungkyunkwan University, Suwon, Korea, and the M.S. and Ph.D. degrees from Seoul National University, Seoul, Korea, in 1978, 1980, and 1987, respectively, all in electrical engineering. From 1990 to 1991, he was with the Department of Electrical Engineering, University of Tennessee, Knoxville, TN, USA, as a Visiting Professor. Since 1988, he has been a Member of the Faculty of Sungkyunkwan University, where he is a Professor with the College of Information and Communication Engineering. His research interests include power electronics of electric machines, electric/hybrid vehicle drives, and power converters for renewable energy systems. Dr. Won was the President of the Korean Institute of Power Electronics, Seoul, Korea, in 2010. Since 2011, he has been a Director of the Korean Federation of Science and Technology Societies, Seoul, Korea. He is the Director of the Samsung Energy Power Research Center, Seoul, Korea. 